\title{
Development of a Feedback Training Protocol and Clinical Force Measuring Walker for Older Patients with Restricted Upper Extremity Weight Bearing
}

\author{
Ansel LAPIER* \\ *Eastern Washington University \\ 310 N. Riverpoint Blvd, Box T, Spokane, Washington, 99202 USA \\ E-mail: lapierlab233@gmail.com
}

\begin{abstract}
Patients recovering from bone disruption due to trauma or surgery need to limit movement to minimize shear force, thereby protecting callus formation and osteogenesis. Patients often use their arms to assist with functional activities, but pushing is frequently limited to $<10 \mathrm{lb}(4.5 \mathrm{~kg})$. With only verbal instructions, patients' ability to limit weight bearing (WB) force accurately is poor. A therapeutic intervention to improve patient adherence with upper extremity (UE) WB guidelines during functional mobility using an instrumented walker could be beneficial. Therefore, the purpose this article is to describe a feedback training protocol to improve ability to modulate weight bearing force in older adults and then provide an overview of the efficacy of this protocol and subsequent development of a Clinical Force Measuring Walker. An instrumented walker was used to measure UE WB during functional mobility in healthy older subjects $(\mathrm{n}=30)$ before, during, and after (immediately and 2 hours) a session of visual and auditory concurrent feedback training. During feedback training, force was significantly reduced with all 3 sessions as compared to baseline. When using the front wheeled walker, UE WB force during the second and third feedback training trials went down compared to the first trial. Force during the third feedback training trial was greater than the two previous trials while transferring sit-to-stand and stand-tosit. After completion of practice with feedback, UE WB force was significantly reduced and remained so 2 hours later. These findings suggest that feedback training is effective for helping patients to modulate UE WB. Use of an instrumented walker and feedback training would be beneficial in clinical practice, especially with older patients. A more intensive feedback training with additional trials and or simultaneous visual and auditory cues during whole practice may be needed to get UE WB below a $10 \mathrm{lb}$ threshold.
\end{abstract}

Keywords: Median sternotomy, Ossification, Cardiac surgery, Rehabilitation, Functional mobility, Bone fracture, Assistive device, Feedback training, Sternal precautions, Instrumented walker

\section{Introduction}

Patients recovering from upper body bone disruption due to trauma or surgery often need to limit use of their upper extremities (UE) during the healing process (Tuyl, Mackney, and Johnson, 2012; Balachandran, et al., 2016; Brocki, Thorup, and Andreasen, 2010). This restriction is thought to minimize shear force and movement between the bone halves to protect callus formation and osteogenesis (Tuyl, Mackney, and Johnson, 2012; Balachandran, et al., 2016; Brocki, Thorup, and Andreasen, 2010; Casha, et al., 2014; Kostenuik and Mirza, 2017; Balachandran, et al., 2014). Common patient diagnoses that require post-fracture (iatrogenic or traumatic) bone ossification include cardiac surgery via median sternotomy, total shoulder arthroplasty, and UE bone fractures (Kostenuik and Mirza, 2017). For example, median sternotomy is commonly performed to access the heart during a variety of surgeries such as coronary artery bypass, heart valve replacement, heart transplantation, and thoracic trauma repairs. The procedure entails sawing longitudinally from the sternal notch to the xiphoid process, separating the sternum with retractors, and wiring the sternal halves together after surgery completion (Zubair and Smith, 2017; Alhalawani and Towler, 2013). Complications can occur when the 
LaPier, Feedback Training with a Force Measuring Walker (2021)

bone halves do not heal correctly, including deep wound infection (osteomyelitis), bony nonunion/instability, and bone dehiscence (Tuyl, Mackney, and Johnson, 2012; Balachandran, et al., 2016; Brocki, Thorup, and Andreasen, 2010; Casha, et al., 2014; Kostenuik and Mirza, 2017).

Restricting arm weight bearing (WB) force to $<10 \mathrm{lb}(4.5 \mathrm{~kg})$ is a common precaution during post-fracture bone ossification. It is difficult to function independently with such significant limitations, especially for older adults (Graham and Brown, 2011; Min, et al., 2015). Aging is associated with frailty and prolonged functional recovery following surgery (Graham and Brown, 2011; Guimaraes and Filho, 2011). Restricting arm use is particularly problematic for patients who need assistance sitting down or standing up from a chair and/or need to use a walker for ambulation. Loss of functional independence can contribute to increased time in the hospital and a greater need for assistance and rehabilitation after discharge (Min et al., 2015; Stocicea, et al., 2017; Edgerton, et al., 2013; Graham and Brown, 2011).

Therefore, appropriate arm use is important for timely return to function. Little is known about how much UE WB force occurs when older patients attempt to use $<10 \mathrm{lb}$, so their ability to safely resume activity and use of a walker is unknown. Previous studies reported that force when using a single arm to assist with standing up from a bench was 27.5 $\mathrm{lb}(12.5 \mathrm{~kg}$ ) (Adams, et al., 2006) and while moving from side-lying to sitting in a bed was $22.2 \mathrm{lb}$ (10.1 kg) (Swanson and Kinney LaPier, 2014). In addition, when older adults use a walker without instructions to limit UE WB, they use on average $22-28 \mathrm{lb}(10.0-12.7 \mathrm{~kg})$, and that force put through the UE varies from $13-40 \%$ of body weight during a gait cycle (Po-Chen and Cherng, 2012; Ishikura, 2001). We previously reported that that with only verbal instructions regarding UE WB, patients are not good at limiting it to $>10 \mathrm{lb}$ during functional mobility (LaPier and Cleary, 2019). Studies examining compliance with touch down weight bearing (defined as $<25 \mathrm{lb}$ ) in patients with lower extremity trauma have found that placing too much weight through the involved side is common (Raaben, et al., 2018; Ruiz, et al., 2014). Hustedt and colleagues instructed healthy subjects to use touch down weight bearing with axillary crutches and found that weight was often up 2.5 times greater than that prescribed (Hustedt, et al., 2012a; Hustedt, et al., 2012b). We have also established that feedback training (FT) can be useful for improving ability to modulating UE WB during functional mobility. Studies employing FT have shown efficacy of its use when leg WB is limited (Raaben, et al., 2018; Hustedt, et al., 2012a; Hustedt, et al., 2012b). Therefore, the purpose of this article is to describe a feedback training protocol designed to improve patients' ability to modulate WB force in older adults and then provide an overview of its efficacy and subsequent development of a Clinical Force Measuring (CFM) Walker.

\section{Methods}

\subsection{Subjects}

This prospective study used a within-subjects design with repeated measures. Subjects $(n=30)$ were a convenience sample who were: 1) healthy adults between the ages of 60-85 years, 2) able to complete Timed Up and Go Test in $<14$ sec, and 3) able to provide informed consent. Exclusion criteria included: 1) recent ( $<6$ months) significant medical event (i.e., stroke, myocardial infarction), 2) pain exacerbated with UE movements/activities, and 3) any contraindication for exercise participation as outlined by the American College of Sports Medicine Guidelines for Exercise Testing (Riebe, et al., 2018). This research project was reviewed and approved by the University's Institutional Review Board.

\subsection{Force Measurements}

Weight bearing force through the UE was measured using an instrumented walker. Force dynamometers (Jamar Smart, Performance Health, Chicago, IL) were wirelessly connected to tablets (Fire HD 10 Tablet, 1080p Full HD, Amazon, Seattle, WA) which interfaced with an application (Jamar Smart, Performance Health, Chicago, IL) that allowed continuous force data collection for up to $30 \mathrm{sec}$. The dynamometers were attached to the grip holds of a standard walker frame (Deluxe Two Button Folding Walker Drive, No. 10200-1, Drive Medical, Post Washington, NY) using platform attachments (Platform Walker/Crutch Attachment No. 10105-1, Drive Medical, Post Washington, NY) and $2.5 \mathrm{~cm}$ Ubolts. The front legs of the walker were replaced with wheeled legs (Universal 5" Walker Wheels, Drive Medical, Post Washington, NY) for the front wheeled walker trials. The front legs of the walker could also be replaced with extension legs (Tall Extension Legs, Drive Medical, Post Washington, NY) to accommodate subjects up to $200 \mathrm{~cm}$ tall. During sitting and standing trials, the instrumented walker was turned backward and placed behind a stool to simulate chair 
LaPier, Feedback Training with a Force Measuring Walker (2021)

armrests. This configuration created a seat height of $46 \mathrm{~cm}$ and armrest height of $60 \mathrm{~cm}$ (see Figure 1.1). During feedback training, an adjustable height table was used to position the visual feedback display (see Figure $1.2 \& 1.3$ ) for subjects to see, and a manual buzzer was used when force exceeded $10 \mathrm{lb}$.

\subsection{Procedures}
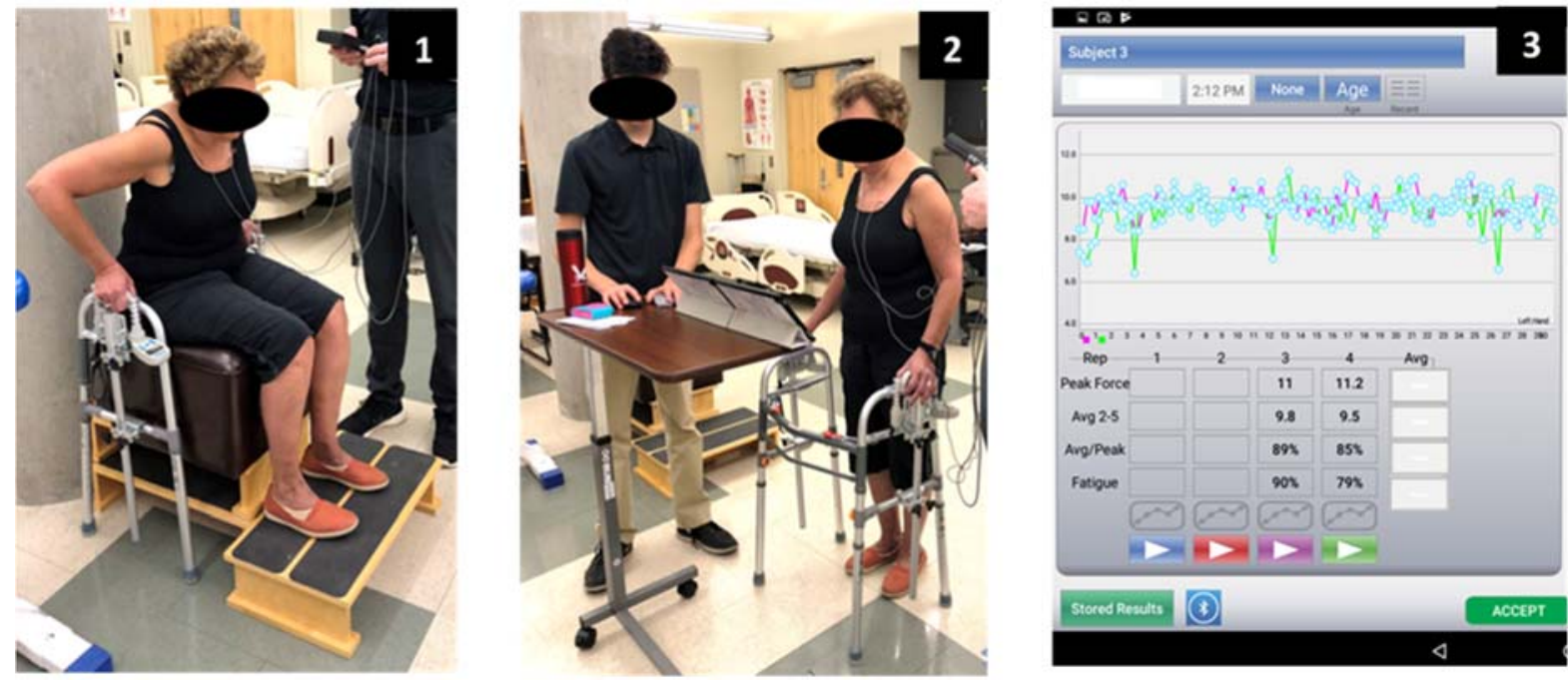

Fig. 1 Force measuring instrumentation. 1) Configuration for sit-stand transfer testing. 2) Configuration for static standing feedback training. 3) Example visual force output screen used during feedback training.

After obtaining informed consent, subjects underwent a basic health history and physical examination to obtain baseline physiological data and information on their health. The Timed Up and Go Test was used as a safety screen for balance impairment; subjects were instructed to stand up from a chair, walk to and around a cone 3 meters away, and return to sitting in the chair. A time of greater than $14 \mathrm{sec}$ indicates an increased risk of falling and was used as an exclusion criterion (Kojima, et al., 2015).

The testing procedures were explained to the study participant. Data collection took place during 4 functional mobility tasks, which included: 1) ambulation using a standard walker, 2) ambulation using a front wheeled walker, 3) standing up from a chair, and 4) sitting down in a chair. For each functional mobility task testing took place: pre-feedback training (self-selected), during 8 feedback training trials (see Figure 2), post-feedback training, and 2 hour follow-up. Order of functional tasks during data collection was randomized and subsequent testing took place in the same order. All trials of walking with an assistive device included a minimum of 5 steps and all trials of transferring from a chair included 3 repetitions of the movement. Testing was stopped if a subject experienced any pain or was unable to perform the task safely (i.e. with proper form, without loss of balance).

At baseline (pre-FT) subjects were only given verbal instruction to use less than $10 \mathrm{lb}$ without any additional cues so the movement strategy was self-selected. During both walker ambulation trials, subjects were instructed how to use the walker and to continue walking until told to stop. The 2 nd through 4 th gait cycles were used for data analysis. During both transfer trials, subjects were instructed to use their arms but to push with less than 10 pounds of pressure while standing up and sitting down 3 times.

Next, subjects participated in an intervention using a FT protocol that is outlined in Figure 2. The feedback protocol included practice for $30 \mathrm{sec}$ as follows: 1) visual feedback during static standing, 2) auditory feedback (buzzer) while ambulating with the standard and the front wheeled walker, 3) visual feedback during static sitting, and 4) auditory feedback (buzzer) during sit-to-stand transfers. Feedback was standardized and consisted of visual force tracing (Fig, 1.C) on a tablet screen and buzzer that was activated when force exceeded $10 \mathrm{lb}$. Peak force during each phase of the feedback training protocol was recorded. Order of the walker ambulation vs transfer FT was randomized. 


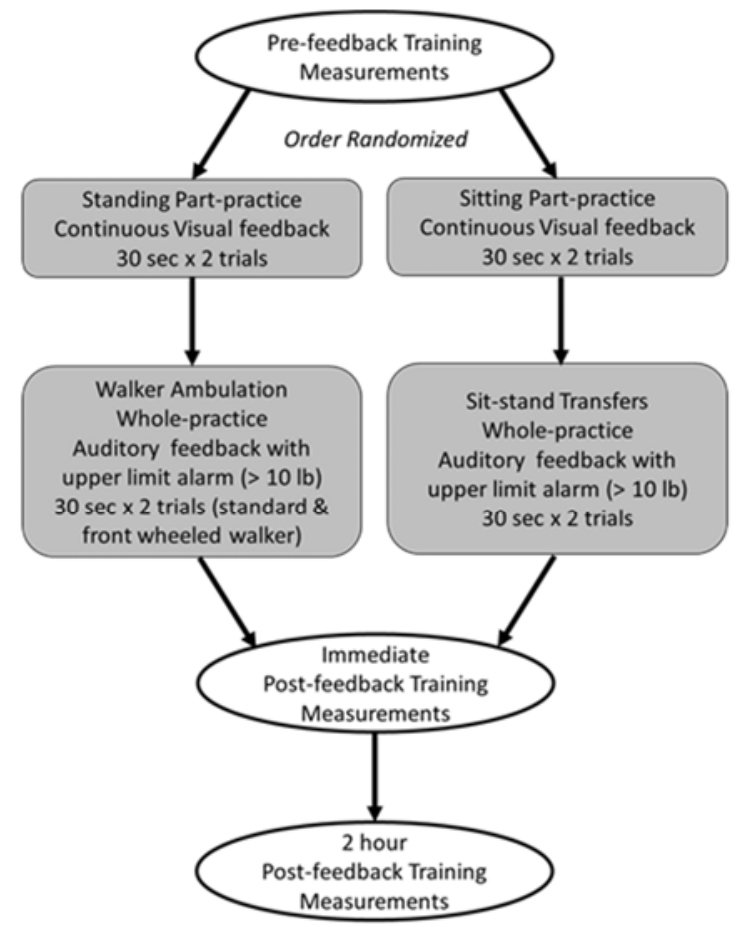

Fig. 2 Flowchart of the feedback training protocol and testing order.

Both visual and auditory feedback were utilized to provide participants with information regarding force placed through their UE. A recent systematic review examining the efficacy of feedback for improving gait parameters found that visual (60\%) and auditory (40\%) feedback were most commonly employed (Chamorro-Moriana, Moreno, and Sevillano, 2018). In this study, a combination of both visual (force output on tablet screen) and auditory feedback was given to subjects. This feedback was provided concurrently (during practice of the skill) as opposed to terminally (after practice of the skill) to best facilitate acquisition of a novel skill. Study participants were first given prescriptive (information on exactly how much weight was being placed through the walker) visual feedback while statically practicing UE WB in standing or sitting. Then, they were given descriptive (buzzer was sounded if force exceeded the $10 \mathrm{lb}$ limit) auditory feedback while practicing the whole task, either walker ambulation and sit-stand transfers (Chamorro-Moriana, Moreno, and Sevillano, 2018). This method facilitated part-practice first followed by wholepractice, a commonly employed principle of motor learning (Sawyer, et al., 2012).

\subsection{Statistical Analyses}

Descriptive statistics for subject demographic variables and UE WB force were calculated. To determine differences in UE WB measurements among the testing conditions, 1-way ANOVA and post hoc tests were used. Differences among the trial were determined using Tukey's Honest Significant Difference. The alpha level was set at $<0.05$. Statistical analyses were performed using Excel ToolPak (Microsoft Corporation, Redmond, WA).

\section{Results and discussion}

\subsection{Results}

The participants $(\mathrm{n}=30)$ in this study had a mean $( \pm \mathrm{SD})$ age of $68.6( \pm 6.4)$ years and $53 \%$ were men. Their mean height, weight, and Body Mass Index were $171.2( \pm 10.0) \mathrm{cm}, 84.4( \pm 18.1) \mathrm{kg}$, and $28.2( \pm 5.8) \mathrm{kg} / \mathrm{m}^{2}$, respectively. Mean time to complete the Timed Up and Go was $7.3(+1.4)$ seconds. Subjects' mean resting vital signs were: heart rate 72 $( \pm 10) \mathrm{bpm}$, systolic blood pressure $126( \pm 16) \mathrm{mm} \mathrm{Hg}$, and diastolic blood pressure $77( \pm 7) \mathrm{mm} \mathrm{Hg}$. On average subjects reported $2.8( \pm 1.8)$ comorbidities. 
LaPier, Feedback Training with a Force Measuring Walker (2021)
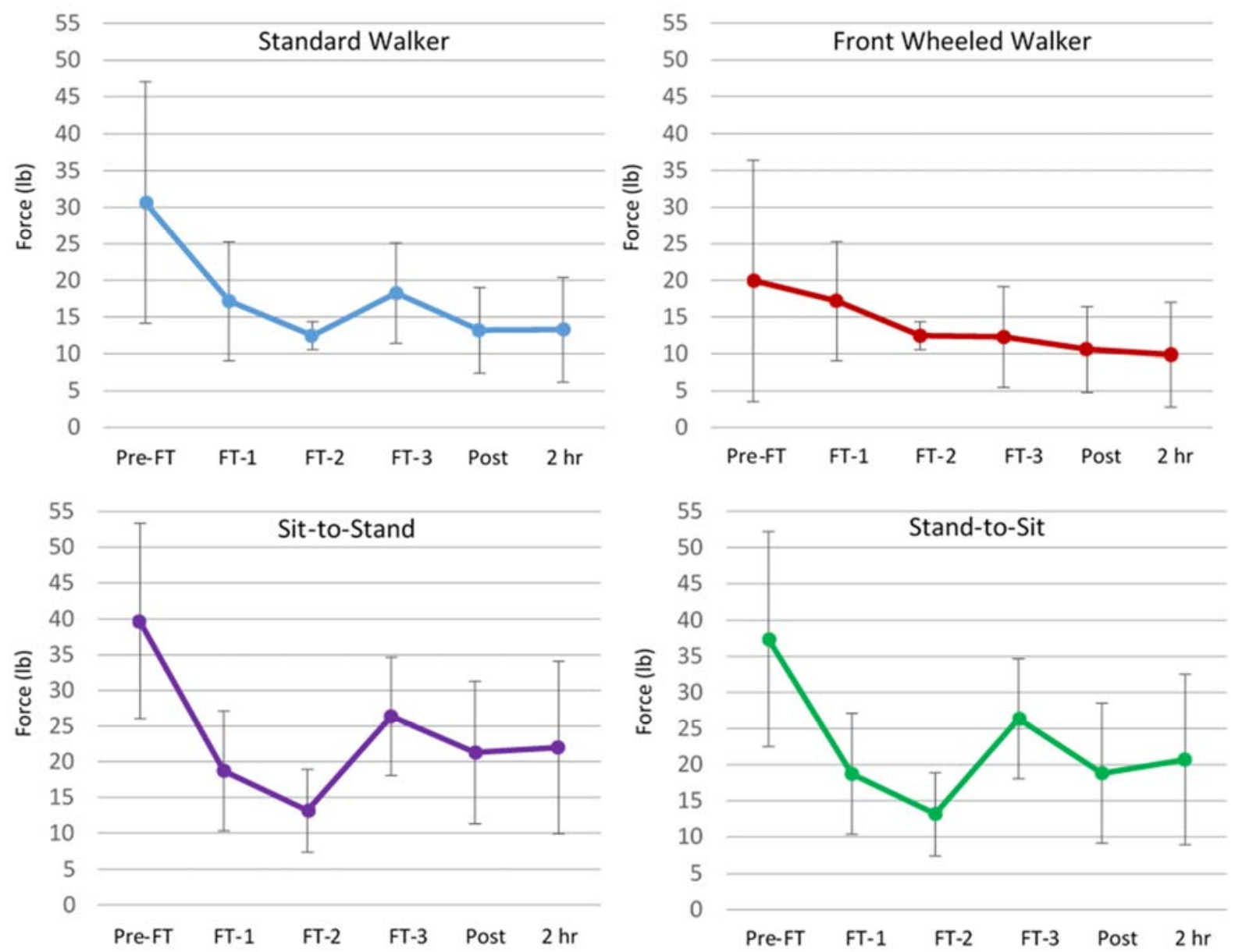

Fig. 3 Force data prior to feedback training (Pre-FT), during 3 trials of feedback training (FT-1, FT-2, FT-3), immediately after feedback training (Post), and 2 hours ( 2 hr) after feedback training.

Serial UE WB force measurements for all 4 functional mobility tasks are shown in Fig. 3. During FT, force was significantly reduced with all 3 sessions as compared to baseline (pre-FT). When using the front wheeled walker, force during the second and third FT trials went down compared to the first FT trial. Force during the third FT trial was greater than the two previous trials while transferring sit-to-stand and stand-to-sit. After completion of all FT practice trials, UE WB force was significantly reduced and remained so 2 hours later.

\subsection{Feedback Training Protocol}

The results of this study demonstrate that a brief feedback intervention can effectively reduce UE WB force exerted during both ambulation with an assistive device and sit-stand transfers. After FT, UE force was reduced during all functional tasks by 46-56\%. But average UE WB for all 4 functional tasks was still greater than the 10 lb limit typically prescribed with sternal precautions. Although other studies (LaPier and Cleary, 2019; Raaben, et al., 2018; Hustedt, et al., 2012a; Hustedt, et al., 2012b) have demonstrated that concurrent visual feedback training is effective at modulating force when WB is limited, study results suggest that a more intensive feedback intervention (i.e., longer practice) and or additional practice sessions would be ideal for older patients recovering from median sternotomy. It also may be helpful to have instrumentation that allows simultaneous visual and auditory feedback while walking.

Study results also demonstrated that the immediate improvements in UE WB are retained in the short term. Force during all functional tasks was not significantly different immediately post-FT or with repeat testing 2 hours later. Hustedt and colleagues (Hustedt, et al., 2012c) also found 2-4 hour retention of lower extremity touch down weight bearing 
LaPier, Feedback Training with a Force Measuring Walker (2021)

compliance after FT. In addition, they also found no significant differences in lower extremity force immediately after FT, 6-8 hours later, and 22-24 hours later, suggesting that skill retention of even a single session may last up to 24 hours (Hustedt, et al., 2012c).

Overall, our findings suggest that use of an instrumented walker and FT would be beneficial in clinical practice, especially to help older patients more accurately follow WB instructions. Most previous studies have used bathroom scales (Hustedt, et al., 2012a; Hustedt, et al., 2012b), force plates/pressure sensing mats (Ishikura, 2001; Anglin and Wyss, 2000), or foot pressure sensors (Raaben, et al., 2018; Hustedt, et al., 2012b; McQuade, Finley, and Oliveira, 2011) to measure WB through the lower extremities. Others have described use of an instrumented walker to measure force placed through the assistive device, but commonly this was accomplished with sensors placed in the walker legs and not the handles (Po-Chen and Cherng, 2012; Fast, et al., 1995). The instrumented walker used in this study had force transducers incorporated into the handles vs. vertical walker legs, which Khodadadi et al found results in easier installation and less measurement error (Khodadadi, et al., 2018). An inexpensive, lightweight walker without bulky addons for the measurement and display of force would have multiple clinical applications for providing feedback to patients who need to limit WB through the upper or lower extremities (Raaben, et al., 2018; Ruiz, et al., 2014). Currently, there are no walkers for use with patients to provide UE WB feedback. The device we previously fabricated using handgrip dynamometers to measure UEWB was only appropriate for research purposes because it was bulky, had remote force displays, and was expensive. Therefore, a method to objectively measure UEWB while patients use a walker in clinical settings is needed.

\subsection{Factors Influencing UE WB}

Previously, we examined differences in UE WB between functional mobility tasks under normal conditions and while wearing a walking boot (LaPier and Cleary, 2021a). Prior to feedback training both with and without the walking cast, subjects used significantly less UE force during front wheeled walker ambulation than other functional tasks. While wearing a walking cast, UE force was increased during front wheeled walker and stand to sit transfers. This suggested that patients with leg impairments may be at risk for using excessive UE WB. We also found that peak force between right and left ranged from 0.2 to $3.5 \mathrm{lb}(0.1$ to $1.6 \mathrm{~kg})$ (LaPier and Cleary, 2021a). There were no significant differences between the left and right sides during both baseline and walking cast trials,. Therefore, UE WB seems to be symmetrical under most circumstances.

We also found that several patient factors are related to UE WB during functional mobility. Handgrip strength, a well-established prognostic indicator of frailty (Graham and Brown, 2011), was directly associated $(r=0.56)$ with UE WB force during transfers and standard walker ambulation. Moderate relationships were found between functional mobility and measurements of balance, quality of life, and Body Mass Index (LaPier and Cleary, 2021a). These measurements might be helpful clinically to identify patients susceptible to using UE WB greater than $10 \mathrm{lb}$ during functional mobility tasks, and therefore determine who would benefit most from postoperative FT with an instrumented walker.

Age also influences the ability to modulate UE WB and response to FT. We found significantly greater UE WB in older $(n=39)$ vs younger $(n=26)$ subjects during functional tasks at baseline, and in both popultons force exceeded that generally recommended $(<10 \mathrm{lb}$ ) during post-fracture ossification (LaPier and Cleary, 2021b). After FT, both groups improved their ability to reduce arm WB force, but the older cohort on average still exceeded the $10 \mathrm{lb}$ limit while the younger cohort did not. Walking with a front wheeled walker produced less arm WB force than walking with a standard walker, which supports the general clinical consensus that a front wheeled walker is best for patients after median sternotomy Tuyl, Mackney, and Johnson, 2012; Brocki, Thorup, and Andreasen, 2010). We found that UE WB force was greater during transfers than walker ambulation. The older cohort used more force during sit-to-stand than stand-to-sit whereas the younger cohort used similar force during both transfer phases. Perhaps older adults need more arm assistance with the concentric sit-to-stand portion than the eccentric stand-to-sit portion of transfers. Several studies have demonstrated that during sit-stand transfers older adults with global weakness have altered motor planning strategies associated with trunk kinematic changes such as reduced maximum angular velocity and larger angular range (Hassani, et al., 2015; van Lummel et al., 2018). 
LaPier, Feedback Training with a Force Measuring Walker (2021)

\subsection{Clinical Force Measuring Walker Prototype Development}

Since our original instrument walker was designed for laboratory testing and not appropriate for use with patients in clinical settings, a prototype CFM Walker was fabricated taking into account common critical care medical equipment used with hospital patients (Bonzino and Peterson, 2015; Paz and West, 2014). Next, a qualitative research methodology was used to garner feedback from rehabilitation professionals. The CFM Walker prototype had externally mounted force transducers wirelessly connected to tablets housed in waterproof cases mounted directly to the walker with multi-planar adjustable mounting arms. It also had: 1) a plate attached below the left lateral support to suspend a chest tube reservoir tank, 2) color-coded adjustable height walker legs, 3) a mounting bracket positioned on the lower front horizontal walker support to hold a portable oxygen tank, 4) ergonomic soft handle grips, 5) a rotating hook mounted below the right lower lateral for a urinary collection bag, and 6) a hook attached directly to the right lower lateral support to tether a Foley catheter.

Using a qualitative description methodology (phenomenology), study participants (rehabilitation professionals) were asked a number of open-ended questions to garner feedback on the walker prototype (Neergard, et al., 2009). The interviews were video recorded and answers transcribed for data analysis. The 5 overarching themes that emerged during data analysis subsequently guided specific alterations to the CFM Walker prototype:

1. The subjects overwhelmingly expressed that a force measuring walker would be very clinically useful with a variety of patients, particularly those recovering from open heart surgery.

2. Integrating the force measuring mechanism into the walker handles / structure would be optimal for arm biomechanics and maneuverability in narrow spaces.

3. Simplifying the force output display and adding upper limit visual and auditory alarms would be easier for patients and rehabilitation professionals to know when UE WB exceeds $10 \mathrm{lb}$.

4. The optimal leg combination was unanimously front 5 " single plane wheels and back standard legs.

5. Subjects also identified several revisions to improve the medical equipment attachments (oxygen tank bracket, Foley Catheter S-hook, oximeter holder).

Using the qualitative data, extensive revisions were made to the CFM Walker prototype (see Figure 4). Thin-film force resistors $(1.8 \times 1.8 \mathrm{~cm})$ were placed under the original walker handgrips (Figure 4.1) and connected to an Arduino System. A visual display with 3 different colored LEDs which were triggered as follows: the green LED was always on, the yellow LED was activated when force was $>7 \mathrm{lb}$, and the red LED was activated when force was $>10 \mathrm{lb}$. An auditory alarm that triggered when force exceeded $10 \mathrm{lb}$ was also included (Figure 4.2). The electrical component housing and external power source were positioned on the front of the walker using a multi-planar clamp mount (Fig. 4.3). A fabricated bracket to hold a standard handheld oximeter and was clamped to the right upper vertical walker support (Fig. 4.4). A swivel hook was mounted to the upper right horizontal support to suspend a urinary collection bag (Fig. 4.5). A 90-degree swivel hook was used so urinary collection bags with a parallel or perpendicular suspension hook could be attached to the walker and maintain bag orientation in the same plane as the right walker support frame. By moving this swivel hook to a higher location, the hook to tether the Foley Catheter was no longer needed, and the urinary collection bag would not drag on the floor. A bracket to suspend the chest tube reservoir from the outside of the lower left horizontal walker support was fabricated (Fig. 4.7). The bracket was $7.7 \mathrm{~cm}$ wide to prevent front-to-back movement and keep the chest tube reservoir parallel to the walker side support. Lastly, the color-coding on the walker legs and oxygen tank attachment were removed because the rehabilitation professionals said they were not necessary. The study participants overwhelmingly preferred 5" single-plane wheels on the front with regular legs on the back of the walker.

\subsection{Clinical Force Measuring Walker Engineering Outcomes}

The modified CFM Walker was evaluated based on predetermined engineering design elements, criteria, constraints, and testing protocols outlined in Table 1. Walker drag was tested using 4 types of front wheels (5" single-plane, 5" swivel, 3" single-plane, and 3" swivel). Results showed that with 5" single-plane front wheels walker horizontal pushpull resistance on smooth, solid surface flooring was significantly less than with the other wheels both with and without medical equipment attached. In addition, 3 types of walker back leg glider feet were tested and none improved drag, so the standard feet were retained. 

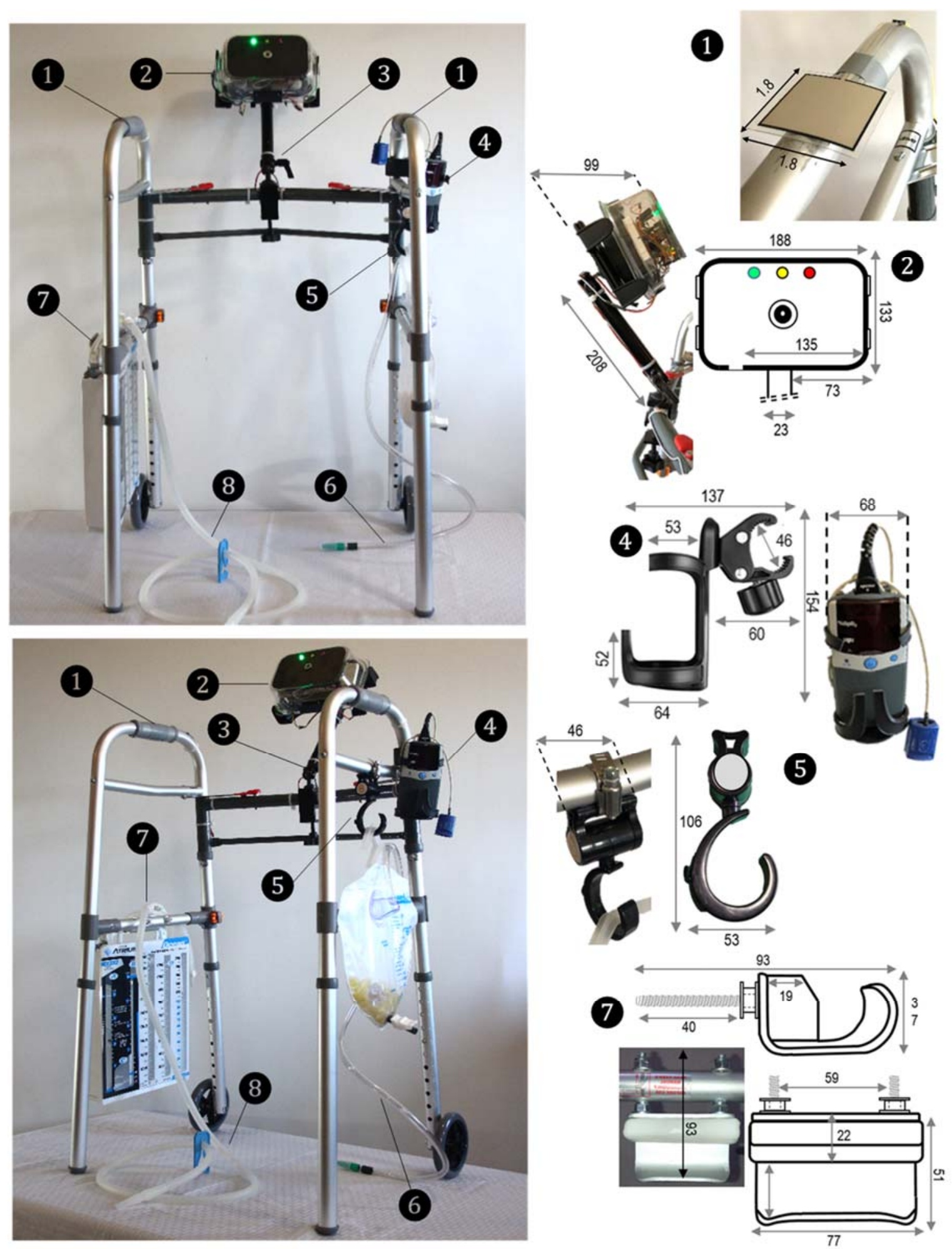

Fig. 4 Components of the Clinical Force Measuring Walker. 1) Thin film force resistors, 2) Feedback display console with Arduino circuit components, 3) Adjustable arm attachment for feedback console, 4) Oximeter holder, 5) Urinary collection bag hook, 6) Foley Catheter, 7) Chest tube reservoir bracket, and 8) Chest tube. All measurements in $\mathrm{mm}$. 
LaPier, Feedback Training with a Force Measuring Walker (2021)

Table 1 Summary of Engineering Design Elements, Criteria, Constraints, and Testing Results.

\begin{tabular}{lll}
\hline Design Elements & Criteria / Constraints & Testing Results \\
\hline $\begin{array}{l}\text { Vertical force } \\
\text { measuring capability }\end{array}$ & Force accuracy $>90 \%$ & Force error rate $=10.3 \%$ \\
\hline Ergonomic handles & Handle diameter $3-6 \mathrm{~cm}$ & Handle diameter $=3.5 \mathrm{~cm}$ \\
\hline $\begin{array}{l}\text { Simple visual \& } \\
\text { auditory feedback } \\
\text { with alarms }\end{array}$ & $\begin{array}{l}\text { Visual \& auditory alarms } \\
\text { detectable at }>1 \mathrm{~m} \text { distance }\end{array}$ & $\begin{array}{l}\text { 1) Visual display }>3 \mathrm{~m} \\
\text { 2) Auditory signal }>3 \mathrm{~m}\end{array}$ \\
\hline $\begin{array}{l}\text { Streamlined, stable, \& } \\
\text { maneuverable frame }\end{array}$ & Frame $<66 \mathrm{~cm}$ wide $\mathrm{x}<63$ deep & $\begin{array}{l}\text { Width }=63.0 \mathrm{~cm} \\
\text { Depth }=50.2 \mathrm{~cm}\end{array}$ \\
\hline $\begin{array}{l}\text { Lightweight } \\
\text { construction }\end{array}$ & Total weight $<6 \mathrm{~kg}$ & $\begin{array}{l}\text { Without medical equipment }=3.9 \mathrm{~kg} \\
\text { With medical equipment }=5.6 \mathrm{~kg}\end{array}$ \\
\hline Minimal drag & Horizontal push-pull resistance & $\begin{array}{l}\text { Without medical equipment }=\text { push } 0.5 \\
\text { kg \& pull } 0.9 \mathrm{~kg} ; \text { With medical } \\
\text { equipment }=\text { push } 0.8 \mathrm{~kg} \& \text { pull } 1.2 \mathrm{~kg}\end{array}$ \\
\hline $\begin{array}{l}\text { Adjustable height } \\
\text { handles }\end{array}$ & $\begin{array}{l}\text { Handle height for patients } \\
1.6-1.8 \mathrm{~m} \text { tall }\end{array}$ & $\begin{array}{l}\text { Shortest to tallest patient height }= \\
1.5-2.0 \mathrm{~m}\end{array}$ \\
\hline Ability to disinfect & Components nonporous \& water & $\begin{array}{l}\text { 1) Nonporous } \rightarrow \text { Yes } \\
\text { resistant }\end{array}$ \\
\hline Affordable cost & Cost $<\$ 500$ & Cost $=\$ 238$ \\
\hline
\end{tabular}

Table 2 provides a detailed parts list of the components used to fabricate the new CFM Walker. The total cost for the revised CFM Walker was \$238. By removing the externally mounted force transducers and creating a simple force feedback interface using an Arduino system, the cost $(\$ 1,171$ savings) and weight (4.5 $\mathrm{kg}$ reduction) of the CFM Walker were substantially reduced. The CFM Walker met all criteria/constraints and the results are shown in Table 1 .

Table 2 The Clinical Force Measuring Walker Materials and Parts List with Cost Breakdown.

\begin{tabular}{|c|c|c|c|}
\hline Component & Description & Qty & TOTAL \\
\hline $\begin{array}{l}\text { Electrical Interface } \\
\text { Mount }\end{array}$ & Arkon Heavy Duty Tablet Clamp Mount; Arkon Mounts, Arcadia, CA & 1 & $\$ 57.90$ \\
\hline Force Transducers & $\begin{array}{l}\text { Thin Film Pressure Sensor, Flex/Bend Sensor ZD10-100 500g } \\
\text { Resistance-type Force Sensing, Walfront LLC, Lewes, DE }\end{array}$ & 2 & $\$ 21.18$ \\
\hline $\begin{array}{l}\text { Circuit Board \& } \\
\text { Components }\end{array}$ & $\begin{array}{l}\text { Arduino Board Kit ELEGOO UNO Project Super Starter Kit with } \\
\text { Tutorial \& UNO R3 Compatible with Arduino IDE }\end{array}$ & 1 & $\$ 36.99$ \\
\hline Power Source & $\begin{array}{l}\text { V50 Always On External Battery Pack with Dual USB Ports - } \\
\text { 12,800mAh, Voltaic Systems, Brooklyn, NY }\end{array}$ & 1 & $\$ 69.00$ \\
\hline Electrical Housing & $\begin{array}{l}\text { Brilliance Storage 3.2-Cup Food Containers with Lids, BPA Free, } \\
\text { Leak Proof, Clear (4-Pack), Rubbermaid, Atlanta, GA }\end{array}$ & 1 & $\$ 8.67$ \\
\hline $\begin{array}{l}\text { Front Wheeled } \\
\text { Walker }\end{array}$ & $\begin{array}{l}\text { Deluxe Two Button Folding Walker with 5-Inch Wheels, \#10210-1, } \\
\text { Drive Medical, Post Washington, NY }\end{array}$ & 1 & $\$ 31.64$ \\
\hline $\begin{array}{l}\text { Urinary Collection } \\
\text { Bag Hook }\end{array}$ & Swivel Multi-Purpose Hook (2 pack), BabyBubz, Vancouver, BC & 1 & $\$ 4.49$ \\
\hline Hose Clamp 4" & ISPINNER 304 Stainless Steel Adjustable 27-102 mm & 1 & $\$ 0.83$ \\
\hline $\begin{array}{l}\text { Chest Tube } \\
\text { Reservoir Hook }\end{array}$ & $\begin{array}{l}\text { Custom, fabricated with aluminum bracket, bolts/nuts/washers, and } \\
\text { heat moldable plastic sheets, Polly Plastics, Midland, MI }\end{array}$ & 1 & $\$ 8.79$ \\
\hline
\end{tabular}


LaPier, Feedback Training with a Force Measuring Walker (2021)

\section{Conclusions}

This research suggests that patients are not good at estimating arm force $<10 \mathrm{lb}$ and that FT could be effective for helping them to modulate UE WB. This data supports use of an instrumented walker and FT would be beneficial in clinical practice, especially with older patients. Although, more intensive FT with additional trials and or simultaneous visual and auditory cues during whole-practice may prove to be best practice. Ultimately a CFM Walker could improve outcomes for patients recovering from upper body bone fracture (iatrogenic or traumatic) with subsequent osteogenesis such as heart surgery performed via median sternotomy and a variety of orthopedic conditions (Johnson, et al., 2019).

\section{References}

Adams, J., Cline, M.J., Hubbard, M., McCullough, T. and Hartman, J., A new paradigm for post-cardiac event resistance exercise guidelines, American Journal of Cardiology, Vol.97, No.2, (2006), pp.281-286.

Alhalawani, A.M. and Towler, M.R., A review of sternal closure techniques, Journal of Biomaterials Applications, Vol.28, No.4, (2013), pp.483-497.

Anglin, C. and Wyss, U.P., Arm motion and load analysis of sit-to-stand, stand-to-sit, cane walking and lifting, Clinical Biomechanics, Vol.15, (2000), pp.441-448.

Balachandran, S., Lee, A., Denehy, L., Lin, K-Y., Royse, A., Royse, C. and El-Ansary, D., Risk factors for sternal complications after cardiac operations: A systematic review, The Annals of Thoracic Surgery, Vol.102, No.6, (2016), pp.2109-2117.

Balachandran, S., Lee, A., Royse, A., Denehy, L. and El-Ansary, D., Upper limb exercise prescription following cardiac surgery via median sternotomy: A web survey, Journal of Cardiopulmonary Rehabilitation and Prevention, Vol.34, No.6, (2014), pp.390-395.

Bonzino, J. and Peterson D., Medical Devices and Human Engineering (2015), CRC Press.

Brocki, B.C., Thorup, C.B., and Andreasen, J.J., Precautions related to midline sternotomy in cardiac surgery: A review of mechanical stress factors leading to sternal complications, European Journal of Cardiovascular Nursing, Vol.9, No.2, (2010), pp.77-84.

Casha, A.R., Manché, A., Gatt, R., Duca, E., Gauci, M., Schembri-Wismayer, P., Camilleri-Podesta, M-T. and Grima, J.N., Mechanism of median sternotomy dehiscence, Interactive CardioVascular and Thoracic Surgery, Vol.19, No.4, (2014), pp.617-621.

Chamorro-Moriana, G., Moreno, A.J. and Sevillan, J.L., Technology-based feedback and its efficacy in improving gait parameters in patients with abnormal gait: A systematic review, Sensors, Vol.18, No.142, (2018).

Edgerton, J.R., Herbert, M.A., Mahoney, C., Armstrong, D., Dewey, T.M., Holper, E., Roper, K. and Mack, M.J., Longterm fate of patients discharged to extended care facilities after cardiovascular surgery, The Annals of Thoracic Surgery, Vol.96, No.3, (2013), pp.871-877

Fast, A., Wang, F.S., Adrezin, R.S., Cordaro, M.A., Ramis, J. and Sosner, J., The instrumented walker: usage patterns and forces, Archives of Physical Medicine and Rehabilitation, Vol.76, (1995), pp.484-491.

Graham, A. and Brown, C.H., Frailty, aging, and cardiovascular surgery, Anesthesia \& Analgesia, Vol.124, No.4, (2011), pp.1053-1060.

Guimaraes, M.N. and Filho, C., Functional status change in older adults undergoing coronary artery bypass surgery, Sao Paulo Medical Journal, Vol.129, No.2, (2011), pp.99-106.

Hassani, A., Kubicki, A., Brost, V., Mourey, F. and Yang, F., Kinematic analysis of motor strategies in frail aged adults during the timed up and go: How to spot the motor frailty?, Clinical Interventions in Aging, Vol.10, (2015), pp.505513.

Hustedt J.W., Blizzard D.J., Baumgaertner M.R., Leslie M.P. and Grauer J.N., Effect of age on partial weight-bearing training, Orthopedics,. Vol.35, No.7, (2012b), pp.1061-1067.

Hustedt, J.W., Blizzard, D.J., Baumgaertner, M.R., Leslie, M.P. and Grauer, J.N., Is it possible to train patients to limit weight bearing on a lower extremity?, Orthopedics, Vol.35, No.1, (2012a), pp.31-37.

Hustedt, J.W., Blizzard, D.J., Baumgaertner, M.R., Leslie, M.P. and Grauer, J.N., Lower-extremity weight-bearing compliance is maintained over time after biofeedback training, Orthopedics, Vol.35, No.11, (2012), pp.1644-1648.

Ishikura, T., Biomechanical analysis of weight bearing force and muscle activation levels in the lower extremities during gait with a walker, Acta Medica Okayama, Vol.55, No.2, (2001), pp.73-82. 
LaPier, Feedback Training with a Force Measuring Walker (2021)

Johnson, J.K., Lohse, B., Bento, H.A., Noren, C.S., Marcus, R.L. and Tonna, J.E., Improving outcomes for critically ill cardiovascular patients through increased physical therapy staffing, Archives of Physical Medicine and Rehabilitation, Vol.100, No.2, (2019), pp.270-277.

Khodadadi, M., Baniasad, M.A., Arazpour, M., Farahmand, F. and Zohoor, H., Designing instrumented walker to measure upper-extremity's efforts: A case study, Assistive Technology, Vol.26, (2018), pp.1-9.

Kojima, G., Masud, T., Kendrick, D., Morris, R., Gawler, S., Treml, J. and Iliffe, S., Does the timed up and go test predict future falls among British community-dwelling older people? Prospective cohort study nested within a randomised controlled trial, BMC Geriatrics, Vol.15, No.38, (2015).

Kostenuik, P. and Mirza, F.M., Fracture healing physiology and the quest for therapies for delayed healing and nonunion, Journal of Orthopaedic Research, Vol.35, (2017), pp.213-223.

LaPier, A. and Cleary, K., Feedback training improves accuracy of estimating upper extremity weight bearing during functional task - implications after open heart surgery, International Journal of Physiotherapy and Research, Vol.7, No.4, (2019), pp.3163-3172, DOI: 10.16965/ijpr.2019.151.

LaPier, A. and Cleary, K., Feedback training improves compliance with sternal precaution guidelines during functional mobility: Implications for optimizing recovery in older patients after median sternotomy, Applied Bionics and Biomechanics, Vol.2021, (2021a), https://doi.org/10.1155/2021/8889502

LaPier, A. and Cleary, K., The influence of age and feedback training on ability to modulate upper extremity weight bearing force and pectoralis major muscle recruitment while following sternal precautions, Physical Therapy and Rehabilitation, Vol.8, No.1, (2021), doi.org/10.7243/2055-2386-8-1.

McQuade K.J., Finley, M. and Oliveira, A.S., Upper extremity joint stresses during walker-assisted ambulation in postsurgical patients, Rev Bras Fisioter, Vol.15, No.4, (2011), pp.332-337.

Min, L., Mazzurco, L., Gure, T.R., Cigolle, C.T., Lee, P., Bloem, C., Chan, C-L., Romano, M.A., Nallamothu, B.K., Langa, K.M., Prager, R.L. and Malani, P.N., Longitudinal functional recovery after geriatric cardiac surgery, Journal of Surgical Research, Vol.194., No.1, (2015), pp.25-33.

Neergaard, M.A., Olesen, F., Andersen, R.S. and Sondergaard, J., Qualitative description - the poor cousin of health research?, BMC Medical Research Methodology, Vol.9, No.52, (2009).

Paz, J.C. and West, M.P., Acute Care Handbook for Physical Therapists (2014), Elsevier.

Po-Chen, Y. and Cherng, L., Using walker during walker: A pilot study for health elder, Work, Vol.41, (2012), pp.20812085.

Raaben, M., Holtslag, H.R., Leenen, L.P.H., Augustine, R. and Blokuis, T.J., Real-time bisual biofeedback during weight bearing improves therapy compliance in patients following lower extremity fractures, Gait Posture, Vol.59, (2018), pp.206-210.

Riebe, D., Ehrman, J.K., Liguori, G. and Magal, M., ACSM's Guidelines for Exercise Testing and Prescription (2018). American College of Sports Medicine.

Ruiz, F.K., Fu, M.C., Bohl, D.D., Hustedt, J.W., Baumgaertner, M.R., Leslie, M.P. and Grauer, J. N., Patient compliance with postoperative lower extremity touch down weight bearing orders at a level I trauma center, Orthopedics, Vol.37, No.6, (2014), pp.552-556.

Sawyers, A., Hahn, M.E., Kelly, V.E., Czerniecki, J.M. and Kartin, D., Beyond componentry: How principles of motor learning can enhance locomotor rehabilitation of individuals with lower limb loss - a review, Journal of Rehabilitation Research and Development, Vol.49, No.10, (2012), pp.1431-1442.

Stoicea, N., You, T., Eiterman, A., Hartwell, C., Davila, V., Marjoribanks, S., Florescu, C., Bergese, S.D. and Rogers, B., Perspectives of post-acute transition of care for cardiac surgery patients, Frontiers in Cardiovascular Medicine, Vol.27, No.4, (2017), pp.70.

Swanson, L.B. and LaPier, T.K., Upper extremity forces generated during activities of daily living, Journal of Acute Care Physical Therapy, Vol.5, No.2, (2014), pp.70-76.

Tuyl, L.J., Mackney, J.H., and Johnston, C.L., Management of sternal precautions following median sternotomy by physical therapist in Australia: A web-based survey, Physical Therapy, Vol.92, No.1, (2012), pp.83-97.

van Lummel, R.C., Evers, J., Niessen, M., Beek, P.J and van Dieen, J.H., Older adults with weaker muscle strength stand up from a sitting position with more dynamic trunk use, Sensors, Vol.18, (2018), doi:10.3390 / s18041235.

Zubair, M.H. and Smith, J.M., Updates in minimally invasive cardiac surgery for general surgeons, Surgical Clinics of North America, Vol.97, No.4, (2017), pp.889-898. 